PROCEEDINGS OF THE

AMERICAN MATHEMATICAL SOCIETY

Volume 131, Number 5, Pages 1443-1452

S 0002-9939(02)06694-7

Article electronically published on September 5, 2002

\title{
ON APPROXIMATIONS OF RANK ONE $\mathcal{H}_{-2}$-PERTURBATIONS
}

\author{
S. ALBEVERIO, V. KOSHMANENKO, P. KURASOV, AND L. NIZHNIK
}

(Communicated by Joseph A. Ball)

\begin{abstract}
Approximations of rank one $\mathcal{H}_{-2}$-perturbations of self-adjoint operators by operators with regular rank one perturbations are discussed. It is proven that in the case of arbitrary not semibounded operators such approximations in the norm resolvent sense can be constructed without any renormalization of the coupling constant. Approximations of semibounded operators are constructed using rank one non-symmetric regular perturbations.
\end{abstract}

\section{INTRODUCTION}

The theory of finite rank perturbations of self-adjoint operators attracted much attention of physicists and mathematicians, in particular it leads to exactly solvable models 1 1, 4, 5, 17, 18, 19, 20, 21, 22, 29]. This theory has been presented in several review papers and summarized in research monographs [3, 9, 14, 22, 31, 32, 35. The aim of the current paper is to clarify a few additional properties of such perturbations, namely the possibility to construct approximations by operators with regular perturbations. Such approximations can be constructed in many different ways, but approximations in the norm resolvent sense attract special attention, since they can give much information concerning the spectrum of the operator to be approximated. It has been discovered already in 1961 by F.Berezin and L.Faddeev [12] that such approximations of highly singular (of order $\mathcal{H}_{-2}$ ) perturbations of positive operators cannot be constructed without any renormalization of the coupling constant. To construct approximations the authors used ideas from field theory, where renormalization became a standard tool. It has been shown in [7] that approximations of rank one $\mathcal{H}_{-2}$-perturbations of not semibounded operators can be constructed if a certain additional condition on the perturbation is satisfied. This approximation in the strong resolvent sense was constructed without any use of the renormalization technique. In the current paper we show that if the original operator is not semibounded, then any rank one $\mathcal{H}_{-2}$-perturbation can be approximated in the norm resolvent sense. Approximations of semibounded operators by non-self-adjoint rank one regular perturbations are discussed as well.

The paper is organized as follows. In Section 2 we give an introduction into the theory of rank one singular perturbations of self-adjoint operators. The main results are presented in Section 3.

Received by the editors July 19, 2001 and, in revised form, December 7, 2001.

2000 Mathematics Subject Classification. Primary 47A55, 47B25; Secondary 81Q15.

Key words and phrases. Self-adjoint operators, singular interactions.

(C)2002 American Mathematical Society 


\section{RANK ONE PERTURBATIONS AND THE EXTENSION THEORY}

In this section we follow closely the presentation given in [9], where one can find all details and proofs. Rank one perturbations of a self-adjoint operator $A$ acting in the Hilbert space $\mathcal{H}$ are formally given by the expression

$$
A_{\alpha}=A+\alpha\langle\varphi, \cdot\rangle \varphi
$$

where the coupling parameter $\alpha$ is real and the vector $\varphi$ is from the scale of Hilbert spaces associated with the operator $A$

$$
\ldots \subset \mathcal{H}_{3} \subset \mathcal{H}_{2} \subset \mathcal{H}_{1} \subset \mathcal{H} \subset \mathcal{H}_{-1} \subset \mathcal{H}_{-2} \subset \mathcal{H}_{-3} \subset \ldots
$$

The norm in each space $\mathcal{H}_{s}$ is given by $\|u\|_{s}^{2}=\left\langle u,(|A|+1)^{s} u\right\rangle$. The spaces $\mathcal{H}_{2}$ and $\mathcal{H}_{1}$ coincide with the domains of the operator and its quadratic form respectively. See [9, 35], where the geometry of this scale of Hilbert spaces is described in detail.

Rank one perturbations can be characterized by their order. If the vector $\varphi$ belongs to the space $\mathcal{H}_{-s}(A), s \geq 1$, and does not belong to $\mathcal{H}_{-s+1}(A)$, then we say that the perturbation has order $-s$. The vector $\varphi$ has order zero if and only if $\varphi \in \mathcal{H}$. Let us consider perturbations of order $0,-1,-2,-3$, respectively.

Order 0 . If $\varphi \in \mathcal{H}$, then the perturbation operator $\alpha\langle\varphi, \cdot\rangle \varphi$ is bounded and the operator $A_{\alpha}$ is self-adjoint on the domain of the operator $A$. The resolvent of the perturbed operator contains the complete information on its spectrum and is given by

$$
\frac{1}{A_{\alpha}-\lambda}=\frac{1}{A-\lambda}-\frac{1}{1 / \alpha+\left\langle\varphi, \frac{1}{A-\lambda} \varphi\right\rangle}\left\langle\frac{1}{A-\bar{\lambda}} \varphi, \cdot\right\rangle \frac{1}{A-\lambda} \varphi
$$

Order -1 . If $\varphi \in \mathcal{H}_{-1}(A) \backslash \mathcal{H}$, then the perturbation operator is infinitesimally form bounded with respect to the operator $A$ and the perturbed operator is uniquely defined, but its domain is different from the domain of the original operator $A$. The operators $A_{\alpha}$ and $A$ are two (different) self-adjoint extensions of the operator $A_{0}$ being the restriction of $A$ to the domain

$$
\operatorname{Dom}\left(A_{0}\right)=\{\psi \in \operatorname{Dom}(A):\langle\varphi, \psi\rangle=0\} .
$$

The operator $A_{0}$ has deficiency indices $(1,1)$ and any function $\psi$ from the domain of the adjoint operator $A_{0}^{*}$ possesses the following representation:

$$
\psi=\tilde{\psi}+b(\psi) \frac{A}{A^{2}+1} \varphi
$$

where $\tilde{\psi} \in \operatorname{Dom}(A)$ and $b(\psi)$ is a certain complex constant depending on the element $\psi$. Then all self-adjoint extensions of $A_{0}$ can be described as the restrictions of the adjoint operator with the domain

$$
\operatorname{Dom}\left(A_{0}^{*}\right)=\left\{\psi=\tilde{\psi}+b(\psi) \frac{A}{A^{2}+1} \varphi, \tilde{\psi} \in \operatorname{Dom}(A), b(\psi) \in \mathbf{C}\right\}
$$

to the set of functions satisfying the boundary conditions

$$
\langle\varphi, \tilde{\psi}\rangle=\gamma b(\psi)
$$


where $\gamma \in \mathbf{R} \cup\{\infty\}$ is the parameter describing the extension 11 To establish the relations between the extension parameter $\gamma$ and the coupling constant $\alpha$ one can simply apply the linear operator $A_{\alpha}$ to an arbitrary function from the domain of the adjoint operator $A_{0}^{*}$

$$
\begin{aligned}
A_{\alpha} \psi & =(A+\alpha\langle\varphi, \cdot\rangle \varphi)\left(\tilde{\psi}+b(\psi) \frac{A}{A^{2}+1} \varphi\right) \\
& =\left\{A \tilde{\psi}-b(\psi) \frac{1}{A^{2}+1} \varphi\right\}+\left[\alpha\langle\varphi, \tilde{\psi}\rangle+b(\psi)+\alpha b(\psi)\left\langle\varphi, \frac{A}{A^{2}+1} \varphi\right\rangle\right] \varphi
\end{aligned}
$$

The latter formula defines an operator in the Hilbert space $\mathcal{H}$ only if the expression $[\cdot]$ vanishes, i.e. if

$$
\langle\varphi, \tilde{\psi}\rangle=-\left(\frac{1}{\alpha}+\left\langle\varphi, \frac{A}{A^{2}+1} \varphi\right\rangle\right) b(\psi) .
$$

It follows that the parameters $\alpha$ and $\gamma$ are related by

$$
\gamma=-\frac{1}{\alpha}-\left\langle\varphi, \frac{A}{A^{2}+1} \varphi\right\rangle=-\frac{1}{\alpha}-c
$$

where we introduce the parameter $c=\left\langle\varphi, \frac{A}{A^{2}+1} \varphi\right\rangle$, which plays a very important role for perturbations of order -2 . Since the operators $A_{\alpha}$ and $A$ are two extensions of one symmetric operator with deficiency indices $(1,1)$, their resolvents are related by Krein's formula [2, 23, 24]

$$
\frac{1}{A_{\alpha}-\lambda}=\frac{1}{A-\lambda}-\frac{1}{1 / \alpha+c+\left\langle\varphi, \frac{1+A \lambda}{A-\lambda} \frac{1}{A^{2}+1} \varphi\right\rangle}, \lambda \in \rho\left(A_{\alpha}\right) \cap \rho(A) .
$$

This formula describes the spectral properties of the operator $A_{\alpha}$ in terms of the coupling parameter $\alpha$ and Krein's $Q$-function $\left\langle\varphi, \frac{1+A \lambda}{A-\lambda} \frac{1}{A^{2}+1} \varphi\right\rangle$.

Order -2. If $\varphi \in \mathcal{H}_{-2}(A) \backslash \mathcal{H}_{-1}(A)$, then the perturbed operator cannot be uniquely defined by the formal expression. This fact can be explained using different points of view. Here we follow the lines of extension theory. The restricted operator $A_{0}$ determined by the same formula (2.4) is a symmetric operator with deficiency indices $(1,1)$, and the operator $A_{\alpha}$ should be one of its self-adjoint extensions characterized by boundary conditions of the form (2.6). But it is impossible without taking into account any additional assumptions to establish the relation between the extension parameter $\gamma$ and the formal coupling constant $\alpha$. The reason is that the functional $\varphi$ is not a bounded linear functional on $\operatorname{Dom}\left(A_{0}^{*}\right)$ and therefore calculation similar to (2.7) cannot be carried out. One of the possibilities to establish this relation is to extend the functional $\varphi$ from $\mathcal{H}_{2}(A)=\operatorname{Dom}(A)$ to $\operatorname{Dom}\left(A_{0}^{*}\right)$ as a bounded linear functional. All such extensions $\varphi^{c}$ are parameterized by one real parameter $c$ and are determined by [6, 7, 9

$$
\left\langle\varphi^{c}, \tilde{\psi}+b(\psi) \frac{A}{A^{2}+1} \varphi\right\rangle=\langle\varphi, \tilde{\psi}\rangle+c b(\psi) .
$$

The parameter $c$ can be chosen arbitrary real $c \in \mathbf{R}$. Taking into account additional properties of the functional one can sometimes determine the parameter $c$ in a unique way if one requires that the extended functional $\varphi^{c}$ has the same properties as $\varphi$. In [6, 7, 9] this question was discussed in detail supposing that the functional

\footnotetext{
${ }^{1}$ This approach to the extension theory for symmetric (not necessarily semibounded) operators is similar to Birman-Krein-Vishik theory applicable to semibounded operators only (see 11 for a recent review and references).
} 
$\varphi$ and the operator $A$ are homogeneous with respect to a certain group of unitary transformations. In what follows we are always going to suppose that the real parameter $c$ is fixed and therefore the perturbed operator is defined uniquely by the following expression:

$$
A_{\alpha}=A+\alpha\left\langle\varphi^{c}, \cdot\right\rangle \varphi
$$

Then the resolvent of the operator $A_{\alpha}$ is given by (2.10), since the regularized resolvent

$$
Q(\lambda)=\left\langle\varphi, \frac{1+A \lambda}{A-\lambda} \frac{1}{A^{2}+1} \varphi\right\rangle
$$

is well defined for any $\varphi \in \mathcal{H}_{-2}(A)$. This function is a Nevanlinna function, i.e. it is analytic and has positive imaginary part in $\Im \lambda>0$.

Order -3 . If $\varphi \in \mathcal{H}_{-3}(A) \backslash \mathcal{H}_{-2}(A)$, then the restricted operator $A_{0}$ is densely defined but no self-adjoint operator in $\mathcal{H}$ (different from $A$ ) can be associated with the formal expression (2.1). One can construct an operator corresponding to this rank one perturbation using a certain extended Hilbert or Pontryagin space. The approach involving spaces with indefinite metrics was developed in $15,16,34,33$. A realization of the operator $A_{\alpha}$ using Hilbert spaces is given in [27]. Generalizations of the latter approach to general perturbations of order $-n, n \geq 4$, leads to non-self-adjoint operators with real spectrum [26, 28]. Discussions of such high order perturbations are outside the scope of the present article.

In what follows we are going to study rank one perturbations of order -1 and -2 only. Such perturbations are called singular, since the domain of the operator is not invariant under them. The perturbations which are determined by zero order vectors are called regular. The characteristic property of such perturbations is that the domain of the perturbed and original operators coincide. In particular approximations of singular perturbations by regular ones will be discussed. The question of approximating singular perturbations is of particular interest for perturbations of order -2 , since the perturbed operator is not uniquely defined in this case.

\section{Approximations of RANK one Singular PERTURbations}

In this section we are going to discuss approximations of rank one perturbations in the norm resolvent sense. Consider a sequence of operators with bounded rank one perturbations

$$
A_{\alpha}^{n}=A+\alpha\left\langle\varphi_{n}, \cdot\right\rangle \varphi_{n}, \quad \varphi_{n} \in \mathcal{H} .
$$

We say that the operator sequence approximates the operator $A_{\alpha}$ in the norm resolvent sense if and only if

$$
\left\|\frac{1}{A_{\alpha}^{n}-\lambda}-\frac{1}{A_{\alpha}-\lambda}\right\| \rightarrow 0 \text { as } n \rightarrow \infty
$$

for any $\lambda, \Im \lambda \neq 0$ If $\varphi \in \mathcal{H}_{-1}$, then such approximations can be constructed choosing any sequence $\varphi_{n} \in \mathcal{H}$ converging to $\varphi$ in the norm of the Hilbert space $\mathcal{H}_{-1}$. Such sequences always exist, since the space $\mathcal{H}$ is dense in $\mathcal{H}_{-1}$ (in the norm $\|\cdot\|_{-1}$ ) [9, 35]. Therefore we are going to discuss the question of how to construct such

\footnotetext{
${ }^{2}$ Note that we are not going to consider the approximations with the renormalization of the coupling constant, i.e. in what follows the real parameter $\alpha$ is always independent of $n$. For approximations with such renormalizations see [12, 20].
} 
approximations only in the case $\varphi \in \mathcal{H}_{-2}$. Approximations of such perturbations in the strong resolvent sense were discussed in detail in [9].

For example the following theorem has been proven in [9] (Theorem 1.4.4).

Theorem 3.1. Let $A$ be a self-adjoint operator and $\varphi$ be a functional from $\mathcal{H}_{-2}(A)$, $\|(1 /(A-i)) \varphi\|=1$. Let $\varphi_{n}$ be any sequence from the Hilbert space converging to $\varphi$ in $\mathcal{H}_{-2}(A)$ and let $\lim _{n \rightarrow \infty}\left\langle\varphi_{n},\left(A /\left(A^{2}+1\right)\right) \varphi_{n}\right\rangle=c$. Then the sequence of selfadjoint operators

$$
A_{\alpha}^{n}=A+\alpha\left\langle\varphi_{n}, \cdot\right\rangle \varphi_{n}
$$

converges to $A_{\alpha}$ in the strong resolvent sense. If

$$
\lim _{n \rightarrow \infty}\left|\left\langle\varphi_{n}, \frac{A}{A^{2}+1} \varphi_{n}\right\rangle\right|=\infty
$$

the operators $A_{\alpha}^{n}$ converge to the original operator in the strong resolvent sense.

Using this theorem it was shown that if the operator $A$ is not semibounded, then it is possible to construct approximations without any renormalization of the coupling constant, but only if the vector $\varphi$ satisfies a certain additional condition [9] (Theorem 1.4.5) (see also [7]).

Theorem 3.2. Let $E_{\mathbf{R}_{ \pm}} \varphi \in \mathcal{H}_{-2}(A) \backslash \mathcal{H}_{-1}(A)$. Then there exist two sequences $c_{n}, d_{n} \rightarrow \infty$ such that $\varphi_{n}=E_{\left(-c_{n}, d_{n}\right)} \varphi$ determines the sequence of self-adjoint operators $A_{\alpha}^{n}=A+\alpha\left\langle\varphi_{n}, \cdot\right\rangle \varphi_{n}$ involving bounded perturbations of $A$ converging to the perturbed operator $A_{\alpha}=A+\alpha\langle\varphi, \cdot\rangle \varphi$ in the strong resolvent sense.

By $E_{(a, b)}$ we denote the spectral projector onto the interval $(a, b)$ associated with the self-adjoint operator $A$. The advantage of Theorem[3.2] is that the approximating sequence of regular vectors $\varphi_{n}$ is explicitly constructed using the spectral projector of $A$ and the vector $\varphi$ only. If we drop this restriction, then any singular perturbation of any self-adjoint operator which is not semibounded can be approximated even in the norm resolvent sense.

Theorem 3.3. Let the self-adjoint operator $A$ not be semibounded and let $\varphi \in$ $\mathcal{H}_{-2}(A)$. Then there exists a sequence of regular vectors $\varphi_{n} \in \mathcal{H}, \varphi_{n} \stackrel{\mathcal{H}_{-2}}{\rightarrow} \varphi$, such that the sequence of self-adjoint operators $A_{\alpha}^{n}=A+\alpha\left\langle\varphi_{n}, \cdot\right\rangle \varphi_{n}$ involving bounded perturbations of $A$ converges to the perturbed operator $A_{\alpha}=A+\alpha\langle\varphi, \cdot\rangle \varphi$ in the norm resolvent sense.

Proof. Let $\varphi_{n}$ be a sequence of regular vectors (from the original Hilbert space) converging to $\varphi \in \mathcal{H}_{-2}$ in the norm of $\mathcal{H}_{-2}$. Such a sequence always exists, since the original Hilbert space is dense in $\mathcal{H}_{-2}$ [9, 35]. Then the resolvents of the operators $A_{\alpha}^{n}$ and $A_{\alpha}$ for any $\lambda, \Im \lambda \neq 0$ are given by

$$
\begin{gathered}
\frac{1}{A_{\alpha}^{n}-\lambda}=\frac{1}{A-\lambda}-\frac{\alpha}{1+\alpha\left\langle\varphi_{n}, \frac{1}{A-\lambda} \varphi_{n}\right\rangle}\left\langle\frac{1}{A-\bar{\lambda}} \varphi_{n}, \cdot\right\rangle \frac{1}{A-\lambda} \varphi_{n}, \\
\frac{1}{A_{\alpha}-\lambda}=\frac{1}{A-\lambda}-\frac{\alpha}{1+\alpha\left(c+\left\langle\varphi, \frac{1+\lambda A}{A-\lambda} \frac{1}{A^{2}+1} \varphi\right\rangle\right)}\left\langle\frac{1}{A-\bar{\lambda}} \varphi, \cdot\right\rangle \frac{1}{A-\lambda} \varphi,
\end{gathered}
$$

where $c$ is the real parameter parameterizing all possible definitions of the singularly perturbed operator in the case $\varphi \in \mathcal{H}_{-2}(A) \backslash \mathcal{H}_{-1}(A)$ via (2.11) and $c=\left\langle\varphi, \frac{A}{A^{2}+1} \varphi\right\rangle$ 
in the case $\varphi \in \mathcal{H}_{-1}(A)$. Then the difference between the resolvents can be calculated

$$
\begin{aligned}
\frac{1}{A_{\alpha}^{n}-\lambda}-\frac{1}{A_{\alpha}-\lambda}= & -\frac{\alpha}{1+\alpha\left\langle\varphi_{n}, \frac{1}{A-\lambda} \varphi_{n}\right\rangle}\left\langle\frac{1}{A-\bar{\lambda}} \varphi_{n}, \cdot\right\rangle \frac{1}{A-\lambda} \varphi_{n} \\
& +\frac{\alpha}{1+\alpha\left(c+\left\langle\varphi, \frac{1+\lambda A}{A-\lambda} \frac{1}{A^{2}+1} \varphi\right\rangle\right)}\left\langle\frac{1}{A-\bar{\lambda}} \varphi, \cdot\right\rangle \frac{1}{A-\lambda} \varphi
\end{aligned}
$$

To prove that the resolvents of $A_{\alpha}^{n}$ converge to the resolvent of $A_{\alpha}$ in the operator norm it is enough to show that

$$
\left\|\frac{1}{A-z} \varphi_{n}-\frac{1}{A-z} \varphi\right\| \rightarrow 0, \text { as } n \rightarrow \infty
$$

and

$$
\begin{aligned}
\left\langle\varphi_{n}, \frac{1}{A-z} \varphi_{n}\right\rangle & =\left\langle\varphi_{n}, \frac{A}{A^{2}+1} \varphi_{n}\right\rangle+\left\langle\varphi_{n}, \frac{1+z A}{A-z} \frac{1}{A^{2}+1} \varphi_{n}\right\rangle \\
& \rightarrow c+\left\langle\varphi, \frac{1+z A}{A-z} \frac{1}{A^{2}+1} \varphi\right\rangle, \text { as } n \rightarrow \infty,
\end{aligned}
$$

for any $z, \Im z \neq 0$ (and in particular for $z=\lambda, \bar{\lambda}$ ).

The first limit (3.5) holds for any sequence $\varphi_{n}$ converging to $\varphi$ in the norm of $\mathcal{H}_{-2}$. Similarly, for any such sequence the scalar product $\left\langle\varphi_{n}, \frac{1+z A}{A-z} \frac{1}{A^{2}+1} \varphi_{n}\right\rangle$ converges to $\left\langle\varphi, \frac{1+z A}{A-z} \frac{1}{A^{2}+1} \varphi\right\rangle$. Therefore to prove the theorem it is enough to show that there exists a sequence of regular vectors with the following two properties:

$$
\varphi_{n} \stackrel{\mathcal{H}_{-2}}{\rightarrow} \varphi \text { and } \lim _{n \rightarrow \infty}\left\langle\varphi_{n}, \frac{A}{A^{2}+1} \varphi_{n}\right\rangle=c .
$$

The proof is not trivial only if $\varphi \in \mathcal{H}_{-2}(A) \backslash \mathcal{H}_{-1}(A)$. In fact if $\varphi \in \mathcal{H}_{-1}(A)$, then consider any sequence $\varphi_{n} \in \mathcal{H}$ converging to $\varphi$ in the norm of $\mathcal{H}_{-1}(A)$. Such a sequence always exists since $\mathcal{H}$ is dense in $\mathcal{H}_{-1}(A)$. Every such sequence satisfies 3.7).

Now consider the case $\varphi \in \mathcal{H}_{-2}(A) \backslash \mathcal{H}_{-1}(A)$. Let us pick up an arbitrary vector $\psi \in \mathcal{H}_{-2}(A) \backslash \mathcal{H}_{-1}(A)$ having the additional property

$$
E_{\mathbf{R}_{ \pm}} \psi \in \mathcal{H}_{-2}(A) \backslash \mathcal{H}_{-1}(A)
$$

where $\mathbf{R}_{ \pm}$denotes the open half-infinite intervals on the real axis and $E$ is the spectral projector associated with $A$. Such vector $\psi$ always exists, since the operator $A$ is not semibounded. Really, the operators $E_{\mathbf{R}_{+}} A, E_{\mathbf{R}_{-}} A$ are not bounded. Therefore there exist vectors $\psi_{ \pm} \in \mathcal{H}_{-2}\left(E_{\mathbf{R}_{ \pm}} A\right) \backslash \mathcal{H}_{-1}\left(E_{\mathbf{R}_{ \pm}} A\right)$. Then the vector $\psi=\psi_{+}+\psi_{-}$satisfies the condition (3.8). Let us introduce the sequence of real numbers $a_{n}=\left\langle E_{[-n, n]} \varphi, \frac{A}{A^{2}+1} \varphi\right\rangle$. Then there exist real numbers $c_{n}, d_{n} \geq n$ such that

$$
\begin{gathered}
\beta_{n}^{2}=\left\langle E_{\left(n, d_{n}\right)} \psi, \frac{A}{A^{2}+1} \psi\right\rangle>\left|a_{n}-c\right|, \\
\gamma_{n}^{2}=-\left\langle E_{\left(-c_{n},-n\right)} \psi, \frac{A}{A^{2}+1} \psi\right\rangle>\left|a_{n}-c\right| .
\end{gathered}
$$


The sequence of regular vectors $\varphi_{n}$ can be chosen equal to

$$
\varphi_{n}=E_{[-n, n]} \varphi+\varepsilon_{1, n} E_{\left(-c_{n},-n\right)} \psi+\varepsilon_{2, n} E_{\left(n, d_{n}\right)} \psi,
$$

where the numbers $\varepsilon_{1, n}, \varepsilon_{2, n} \in[0,1]$ are given by $\varepsilon_{1, n}=\frac{\sqrt{a_{n}-c}}{\gamma_{n}}, \varepsilon_{2, n}=0$ and $\varepsilon_{1, n}=0, \varepsilon_{2, n}=\frac{\sqrt{c-a_{n}}}{\beta_{n}}$ if $a_{n}-c \geq 0$, and $a_{n}-c \leq 0$, respectively. The following calculations prove that the sequence $\varphi_{n}$ satisfies conditions (3.7)

$$
\begin{gathered}
\left\langle\varphi_{n}, \frac{A}{A^{2}+1} \varphi_{n}\right\rangle=a_{n}-\varepsilon_{1, n}^{2} \gamma_{n}^{2}+\varepsilon_{2, n}^{2} \beta_{n}^{2}=c, \\
\left\|\varphi_{n}-\varphi\right\|_{\mathcal{H}_{-2} \leq} \quad\left\|E_{(n, \infty)} \varphi\right\|_{\mathcal{H}_{-2}}+\left\|E_{(-\infty,-n)} \varphi\right\|_{\mathcal{H}_{-2}} \\
\quad+\left\|E_{(n, \infty)} \psi\right\|_{\mathcal{H}_{-2}}+\left\|E_{(-\infty,-n)} \psi\right\|_{\mathcal{H}_{-2}} \\
\rightarrow \quad 0, \text { as } n \rightarrow \infty .
\end{gathered}
$$

The theorem is proven.

One can easily prove that this theorem is optimal in the sense that the following lemma is valid [6] (Lemma 2.3).

Lemma 3.4. Let $A \geq 0$ and $\varphi \in \mathcal{H}_{-m}(A) \backslash \mathcal{H}_{-1}(A), m \geq 2$. Let $\varphi_{n}$ be any sequence weakly converging to $\varphi$ in $\mathcal{H}_{-m}(A)$. Then the sequence of self-adjoint operators

$$
A_{\alpha}^{n}=A+\alpha\left\langle\varphi_{n}, \cdot\right\rangle \varphi_{n}
$$

converges to $A$ in the strong resolvent sense.

Approximations of not semibounded self-adjoint operators in the strong resolvent sense involving the renormalization of the coupling constant were constructed in 12, 20. Another possibility to construct approximations of singularly perturbed operators is to use non self-adjoint operators. Consider the following family of operators:

$$
B_{\alpha}^{n}=A+\alpha\left\langle\psi_{n}, \cdot\right\rangle \varphi_{n}
$$

where $\psi_{n}, \varphi_{n} \in \mathcal{H}, \alpha \in \mathbf{R}$. Each of these operators is a bounded perturbation of the self-adjoint operator $A$ and their resolvents can easily be calculated

$$
\frac{1}{B_{\alpha}^{n}-\lambda}=\frac{1}{A-\lambda}-\frac{\alpha}{1+\alpha\left\langle\psi_{n}, \frac{1}{A-\lambda} \varphi_{n}\right\rangle}\left\langle\frac{1}{A-\bar{\lambda}} \psi_{n}, \cdot\right\rangle \frac{1}{A-\lambda} \varphi_{n},
$$

where $\lambda \in \rho\left(B_{\alpha}^{n}\right) \cap \rho(A)$. The following slight modification of Theorems [3.1] and 3.3 can be proven.

Theorem 3.5. Let $A$ be a self-adjoint operator and $\varphi$ be a functional from $\mathcal{H}_{-2}(A)$, $\|(1 /(A-i)) \varphi\|=1$. Let $\varphi_{n}$ and $\psi_{n}$ be any two sequences from the Hilbert space converging to $\varphi$ in $\mathcal{H}_{-2}(A)$ and let $\lim _{n \rightarrow \infty}\left\langle\psi_{n},\left(A /\left(A^{2}+1\right)\right) \varphi_{n}\right\rangle=c$. Then the sequence of (non-self-adjoint) operators

$$
B_{\alpha}^{n}=A+\alpha\left\langle\psi_{n}, \cdot\right\rangle \varphi_{n}
$$

converges to $A_{\alpha}$ in the norm resolvent sense. If

$$
\lim _{n \rightarrow \infty}\left|\left\langle\psi_{n}, \frac{A}{A^{2}+1} \varphi_{n}\right\rangle\right|=\infty
$$

the operators $B_{\alpha}^{n}$ converge to the original operator in the norm resolvent sense. 
We do not give a proof here, since it follows the same lines as the proof of Theorem 3.1 The following theorem shows that approximations by non-self-adjoint operators can be constructed even if the original operator is semibounded. It is a generalization of the results obtained in [10, 30].

Theorem 3.6. Let $A$ be an arbitrary self-adjoint operator and let $\varphi \in \mathcal{H}_{-2}(A)$. Then there exist two sequences of regular vectors $\varphi_{n}, \psi_{n} \in \mathcal{H}, \varphi_{n}, \psi_{n} \stackrel{\mathcal{H}_{-2}}{\rightarrow} \varphi$, such that the sequence of (non-self-adjoint) operators $B_{\alpha}^{n}=A+\alpha\left\langle\psi_{n}, \cdot\right\rangle \varphi_{n}$ involving bounded perturbations of $A$ converges to the perturbed operator $A_{\alpha}=A+\alpha\langle\varphi, \cdot\rangle \varphi$ in the norm resolvent sense.

Proof. We are going to prove the theorem in the case where the operator $A$ is semibounded, since Theorem 3.3 covers the opposite case. Moreover if $A$ is bounded, then $\mathcal{H}_{-2}(A)=\mathcal{H}_{-1}(A)=\mathcal{H}$ and the proof is trivial. Therefore without loss of generality we can suppose that $A$ is positive and not bounded.

Again the proof is not trivial only if $\varphi \notin \mathcal{H}_{-1}(A)$. To use Theorem 3.5 one needs to construct two sequences of regular vectors satisfying the following conditions:

$$
\psi_{n}, \varphi_{n} \stackrel{\mathcal{H}_{-2}}{\rightarrow} \varphi \text { and } \lim _{n \rightarrow \infty}\left\langle\psi_{n}, \frac{A}{A^{2}+1} \varphi_{n}\right\rangle=c .
$$

Let us introduce the real sequence $a_{n}=\left\langle E_{[-n, n]} \varphi, \frac{A}{A^{2}+1} \varphi\right\rangle$. Since $\varphi$ does not belong to $\mathcal{H}_{-1}(A)$, then there exists an interval $\left[c_{n}, d_{n}\right]$ outside $[-n, n]$ such that numbers $b_{n}=\left\langle E_{\left[c_{n}, d_{n}\right]} \varphi, \frac{A}{A^{2}+1} \varphi\right\rangle$ could be arbitrarily large (small), in particular satisfying $\left|b_{n}\right|>\left|c-a_{n}\right|$. Then the sequences $\varphi_{n}, \psi_{n} \in \mathcal{H}$ can be chosen equal to

$$
\begin{aligned}
& \varphi_{n}=E_{[-n, n]} \varphi+\varepsilon_{n} E_{\left[c_{n}, d_{n}\right]} \varphi, \\
& \psi_{n}=E_{[-n, n]} \varphi+\delta_{n} E_{\left[c_{n}, d_{n}\right]} \varphi,
\end{aligned}
$$

where the numbers $\varepsilon_{n}$ and $\delta_{n}$ are given by $\varepsilon_{n}=\frac{\left|c-a_{n}\right|^{1 / 2}}{\left|b_{n}\right|^{1 / 2}}, \delta_{n}=\operatorname{sign}\left(c-a_{n}\right) \operatorname{sign} b_{n} \varepsilon_{n}$. It is obvious that $\left|\varepsilon_{n}\right| \leq 1$, and $\left|\delta_{n}\right| \leq 1$ and $\left\langle\psi_{n}, \frac{A}{A^{2}+1} \varphi_{n}\right\rangle=a_{n}+\varepsilon_{n} \delta_{n} b_{n}=c$. Moreover,

$$
\left\|\varphi_{n}-\varphi\right\|_{\mathcal{H}_{-2}} \leq 2\left(\left\|E_{(n, \infty)} \varphi\right\|_{\mathcal{H}_{-2}}+\left\|E_{(-\infty,-n)} \varphi\right\|_{\mathcal{H}_{-2}}\right) \rightarrow 0, \text { as } n \rightarrow \infty .
$$

Similarly one can prove that $\left\|\psi_{n}-\varphi\right\|_{\mathcal{H}_{-2}} \rightarrow 0$, as $n \rightarrow \infty$. Then Theorem 3.5 implies that the operator sequence $B_{\alpha}^{n}$ converges to the operator $A_{\alpha}$ in the norm resolvent sense.

\section{Conclusions}

In this article it was shown that any rank one $\mathcal{H}_{-2}$-perturbation of a not semibounded self-adjoint operator can be approximated (in the norm resolvent sense) by a sequence of operators with regular perturbations of rank one. In the case of semibounded operators, we prove that approximations by not self-adjoint rank one regular perturbations can be constructed. The approach developed in this paper can easily be generalized to include arbitrary finite rank perturbations [8, 9, 13]. In particular the case of point interactions in $\mathbf{R}^{1}$ has recently been treated in [10, 25, 30]. It remains an open question of how to describe approximations of the higher order rank one perturbations described in [16, 26, 27, 28, 33] 34]. 


\section{ACKNOWLEDGMENTS}

The authors thank the referee for valuable remarks leading to an essential improvement of Theorem 3.3. The second and fourth authors express their gratitude to DFG for the financial support of the project 436 UKR 113/43/1. The third author is grateful to the A. von Humboldt Foundation for the support of his research in Germany and to The Royal Swedish Academy of Sciences.

\section{REFERENCES}

1. V.M.Adamyan and B.S.Pavlov, Zero-radius potentials and M. G. Krein's formula for generalized resolvents, (Russian) Zap. Nauchn. Sem. Leningrad. Otdel. Mat. Inst. Steklov. (LOMI) 149 (1986), Issled. Linein. Teor. Funktsii. XV,7-23 (translation in J. Soviet Math., 42 (1988), 1537-1550). MR 87h:47031

2. N.I.Akhiezer and I.M.Glazman, Theory of linear operators in Hilbert space, vol. I,II, Piman, Boston, 1981 (Translation from Russian). MR 83i:47001a; MR 83i:47001b

3. S.Albeverio, F.Gesztesy, R.Høegh-Krohn, and H.Holden, Solvable models in quantum mechanics, Springer, 1988. MR 90a:81021

4. S.Albeverio and V.Koshmanenko, Singular rank one perturbations of self-adjoint operators and Krein theory of self-adjoint extensions, Potential Anal. 11 (1999), 279-287. MR 2000g:47011

5. S.Albeverio and V.Koshmanenko, On form-sum approximations of singularly perturbed positive self-adjoint operators, J. Func. Anal., 169 (1999), 32-45. MR 2000j:47043

6. S.Albeverio and P.Kurasov, Rank one perturbations, approximations and selfadjoint extensions, J. Func. Anal., 148 (1997), 152-169. MR 98g:47011

7. S.Albeverio and P.Kurasov, Rank one perturbations of not semibounded operators, Integr. Equ. Oper. Theory, 27 (1997), 379-400. MR 98k:47025

8. S.Albeverio and P.Kurasov, Finite rank perturbations and distribution theory, Proc. Amer. Math. Soc., 127 (1999), 1151-1161. MR 99m:47012

9. S.Albeverio and P.Kurasov, Singular perturbations of differential operators. Solvable Schrödinger type operators, London Mathematical Society Lecture Note Series, 271, Cambridge University Press, Cambridge, 2000, xiv+429 pp. MR 2001g:47084

10. S.Albeverio and L.Nizhnik, Approximation of general zero-range potentials, Ukrainian Math. J., 5 (2000). MR 2002c: 81035

11. A.Alonso and B.Simon, The Birman-Krein-Vishik theory of selfadjoint extensions of semibounded operators, J. Operator Theory, 4 (1980), 251-270. MR 81m:47038

12. F.A.Berezin and L.D.Faddeev, A remark on Schrödinger equation with a singular potential, Soviet Math. Dokl., 137 (1961), 1011-1014. MR 23:B2345

13. J.Boman and P.Kurasov, Finite rank singular perturbations and distributions with discontinuous test functions, Proc. Amer. Math. Soc., 126 (1998), 1673-1683. MR 98g:47012

14. Yu.N.Demkov and V.N.Ostrovsky, Zero-range potentials and their applications in atomic physics, Plenum, New York, 1988.

15. J.F.van Diejen and A.Tip, Scattering from generalized point interactions using selfadjoint extensions in Pontryagin spaces, J. Math. Phys., 32 (1991), 630-641. MR 92e:81028

16. A.Dijksma, H.Langer, Yu.Shondin, and C. Zeinstra, Self-adjoint operators with inner singularities and Pontryagin spaces, Operator theory and related topics, Vol. II (Odessa, 1997), 105-175, Oper. Theory Adv. Appl., 118, Birkhäuser, Basel, 2000. MR 2001f: 47039

17. F.Gesztesy and B.Simon, Rank-one perturbations at infinite coupling, J. Func. Anal., 128 (1995), 245-252. MR 95m:47014

18. S.Hassi and H.de Snoo, On rank one perturbations of selfadjoint operators, Integral Equations Operator Theory, 29 (1997), 288-300. MR 98k:47027

19. S.Hassi, H.de Snoo, and A.Willemsma, Smooth rank one perturbations of selfadjoint operators, Proc. Amer. Math. Soc., 126 (1998), 2663-2675. MR 98k:47028

20. A.Kiselev and B.Simon, Rank one perturbations with infinitesimal coupling, J. Func. Anal., 130 (1995), 345-356. MR 96e:47012

21. V. Koshmanenko, Towards the rank-one singular perturbations of self-adjoint operators, Ukrainian Math. J., 43 (1991), 1559-1566. 
22. V.Koshmanenko, Singular quadratic forms in perturbation theory, Kluwer, 1999. MR 2001a:47026

23. M.G.Krein, On Hermitian operators whose deficiency indices are 1, C. R. (Doklady) Acad. Sci URSS (N.S.), 43 (1944), 323-326. MR 6:131a

24. M.G.Krein, On Hermitian operators whose deficiency indices equal to one. II, C. R. (Doklady) Acad. Sci URSS (N.S.), 44 (1944), 131-134. MR 6:179a

25. P.Kurasov, Distribution theory for discontinuous test functions and differential operators with generalized coefficients, J. Math. Anal. Appl., 201 (1996), 297-323. MR 97g:46050

26. P.Kurasov, $H_{-n}$-perturbations of self-adjoint operator and Krein's resolvent formula, Research Report N4, Stockholm Univ., 2001; accepted for publication in Integr. Eq. Oper. Theory.

27. P.Kurasov and K.Watanabe, On rank one $H_{-3}$-perturbations of positive self-adjoint operators, Stochastic processes, physics and geometry: new interplays, II (Leipzig, 1999), 413-422, CMS Conf. Proc., 29, Amer. Math. Soc., Providence, RI, 2000. MR 2001j:47012

28. P.Kurasov and K.Watanabe, On $H_{-4}$-perturbations of self-adjoint operators, Operator Theory: Advances and Applications, 126 (2001), 179-196.

29. S.T.Kuroda and H.Nagatani, $\mathcal{H}_{-2}$-construction and some applications, Mathematical results in quantum mechanics (Prague, 1998), 99-105, Oper. Theory Adv. Appl., 108, Birkhäuser, Basel, 1999. MR 2000f:47022

30. L.Nizhnik, On point interactions in quantum mechanics, Ukrainian Math. J., 49 (1997), $1557-1560$.

31. B.S.Pavlov, The theory of extensions, and explicitly solvable models, (Russian), Uspekhi Mat. Nauk, 42 (1987), 99-131. MR 89b:47009

32. B.S.Pavlov, Boundary conditions on thin manifolds and the semiboundedness of the threebody Schrödinger operator with point potential, (Russian), Mat. Sb. (N.S.), 136(178) (1988), 163-177 (translation in Math. USSR-Sb., 64 (1989), 161-175). MR 90g:35120

33. Yu.Shondin, Quantum mechanical models in $R^{n}$ connected with extensions of the energy operator in a Pontryagin space, (Russian) Teoret. Mat. Fiz., 74 (1988), 331-344 (translation in Theoret. and Math. Phys., 74 (1988), 220-230). MR 89f:81042

34. Yu.Shondin, Perturbation of elliptic operators on thin sets of high codimension, and extension theory in a space with an indefinite metric, (Russian), Zap. Nauchn. Sem. S.-Peterburg. Otdel. Mat. Inst. Steklov. (POMI), 222 (1995), Issled. po Linein. Oper. i Teor. Funktsii. 23, 246-292 (translation in J. Math. Sci. (New York) 87 (1997), 3941-3970). MR 97e:47084

35. B.Simon, Spectral analysis of rank one perturbations and applications, in "CRM Proceedings and Lecture Notes", 8 (1995), 109-149. MR 97c:47008

Institute für Angewandte Mathematik, Univ. Bonn, Wegelerstr. 6, 53155 Bonn, Germany; SFB 256 Bonn, BiBos, Bielefeld-Bonn, CERFim, Locarno and USi (SwitzerLAND)

E-mail address: albeverio@uni-bonn.de

Institute of Mathematics, vul. Tereschenkivs'ka, 3, Kyiv, 01601 Ukraine

E-mail address: kosh@imath.kiev.ua

Department of Mathematics, Stockholm University, 10691 Stockholm, Sweden

E-mail address: pak@matematik.su.se

Current address: Department of Mathematics, Lund Institute of Technology, Box 118, 22100

Lund, Sweden

E-mail address: kurasov@maths.1th.se

Institute of Mathematics, Vul. Tereschenkivs'Ka, 3, Kyiv, 01601 Ukraine

E-mail address: nizhnik@imath.kiev.ua 